\title{
OPTIMAL SHAPE OF WATER TOWERS
}

\author{
ANTHONY SULAK* AND \\ ADVISOR: SHAWN D. RYAN ${ }^{\dagger}$
}

\begin{abstract}
Water tanks can be seen in communities all across the country, but how well are they designed? In this work, we study the optimal shape of water tanks to find the fastest emptying time. In particular, we compare emptying the efficiency for different shaped water towers with the same volume, height, and cross-sectional outlet area. We first review a formula for the emptying time as a function of the volume and the tank's height and then compute the emptying time for several specific tank shapes. The question of whether there exists a tank with a minimal emptying time is also considered in the context of prior work. Finally, our added contribution is to fix the volume of a typical water tank and compute the area and the emptying time for different tank shapes, including composites, in order to develop an understanding for how an optimal tank might be designed.
\end{abstract}

Key words. Water towers, Optimal shapes, Emptying efficiency

1. Introduction. Since the dawn of civilization, the retrieval of water has been a fundamental problem for humanity. To address this problem, early humans would be forced to settle near a body of water. Water was primarily used for drinking, sanitation, and watering crops [10]. However, as cities were built and infrastructure became more common, the need for water in each individual building became more apparent along with the rise of the amount of uses of water. Thus, the need to efficiently move water from one place to another quickly arose. One such solution came in the form of an aqueduct in the Roman times. However, the Minoans, who were much before their time in terms of water systems, used aqueducts before the Romans during the Bronze age [2]. The Minoans had aqueducts, a filtration system, harvested rain water, sewage pipes, etc. Specfically, the aqueduct made it possible for humans to live farther away from bodies of water; however, this range remained limited. The logistics of water remains an issue due to the necessity of water for human life. Storing water for immediate use at specific times has become the modern day equivalent of these logistical issues. The most common way to store water is to use a water tower [2].

Water towers are basic water storage devices capable of holding hundreds of thousands to millions of gallons of water at a time. We can think of water towers as simply water tanks that are elevated off the ground. This is done to allow gravity to enhance water pressure in order to get the water where it needs to go [7]. For each foot of the water tower, the tower increases its water pressure by roughly 0.43 pounds per square inch. An essential feature in the present day is the ability of a water tower to hold a day's worth of water in case of emergency. For example during a power outage, the mechanical pumps cannot work and the people must rely on the backup water supply the water tower provides. Water towers are also used during parts of the day when water demand is high to alleviate strain on the system. Usually the busiest time is in the mornings, when people are getting ready for their day. At night when little water is used, the water tower is refilled for the next day [7]. Marshall says that in general, a town gets their water from a well, river, or lake. The water is cleaned at a water treatment plant and pressurized by the pump to allow the water to travel through

\footnotetext{
*Department of Mathematics, Cleveland State University, Cleveland, OH 44115 (tonysulak@yahoo.com).

†Department of Mathematics, Cleveland State University, Cleveland, OH 44115 (s.d.ryan@csuohio.edu).
} 
the pipes. From there, it will either go to the consumers or the water tank depending on the demand [3].

Most water supplies run between 50 to 100 PSI [3], thus requiring most water towers to have a height of about 120 feet to satisfy that requirement. In regions where the land is elevated, the tank will not have to be as high because the natural elevation will act as the tower's height. In general, a water tank can hold approximately $1,000,000$ gallons of water, which is usually enough water for a given town for one day. That is about 50 times as much water as a backyard swimming pool (e.g., 20,000 to 30,000 gallons). Since water towers can be used during higher demand periods, pumps are designed for the size of the average demand and not the peak demand. The combination of the pumps and towers can save a town significant amounts of money [3]. Thus, the optimal shape of the water tower for efficient distribution of its contents in a minimal amount of time has become an interesting problem from a societal prospective. Moreover, the optimal shape of a water tower is the shape that can empty its contents in the shortest time. Equivalently, the optimal shape has the smallest emptying efficiency coefficient as discussed later in Section 2.

As an example, let us say a pumping station averages 500 gallons per minute or 720,000 gallons per day. In the morning the water consumption is much greater than the average, we assume the demand is 2,000 gallons per minute. The cost between a pump that can support 500 gallons per minute compared to 2,000 gallons per minute is huge [3]. Therefore, it is cheaper to build and maintain a water tower to help the pump during the peak times. Thus, the emptying efficiency is the most important quality. In the following sections we show that the emptying efficiency turns out to only depend on the shape of the tank. Therefore, picking the right shape is crucial. Though there are many different and uniquely shaped water tanks we restrict this work to focus on the cone, hemisphere, sphere, cylinder, inverse cone, and composite of these.

The goal of this work is to investigate an optimal shape for a water tank. In Section 2, we begin with a review of results for basic shapes such as a cone, cylinder, or frustum and once a basic understanding for the important quantities is developed we move on to composite tank shapes. To analyze the best tank, we start with a formula for the emptying time $T$ of a tank as posed by Hanin in [8]. We then consider the emptying efficiency of different shaped water tanks and investigate whether a minimal emptying time exists. This provides a brief overview of [8] and highlights some additional details. In Section 3, we provide new insight on the efficiency of composite tanks by calculating the emptying efficiency using piecewise functions and the emptying time for various tanks. Our predictions are consistent with and help to explain observations about the prevalence of certain shaped water tanks in the present day throughout local communities.

2. Mathematical Model. Let $V$ and $H$ be the volume and height of a tank respectfully. We start with the tank filled to the top with liquid. The liquid will eventually exit the tank through a small hole at the bottom with a cross-sectional area $S$. However, we need to know the cross-sectional area $A(h)$ as function of the height of the liquid $h$. Hence $A(h), 0 \leq h \leq H$ will denote cross-sectional area at $h$ (e.g., $A(0)=S$ ). We assume $A(h)$ to be continuous because we need the crosssectional area at any time $t$ and any height $h$. This cannot be done discretely. 


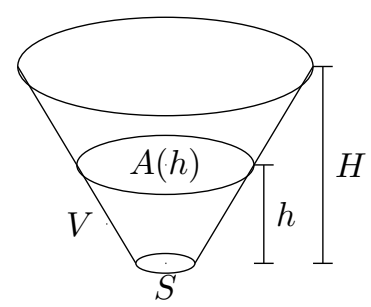

As time passes some small amount $\Delta t$, the height of the liquid will decrease some amount $\Delta h$. We can take the cross-sectional area of the tank at every height drop $\Delta h$ to find an approximation for the volume of the tank. Thus, $\Delta V \approx A(h) \Delta h$. Note that the velocity of the flowing liquid depends on the height of the liquid (i.e., $v=v(h)$ ). Hence, for every time step $\Delta t$ the liquid travels at a velocity $v(h)$ through the area of the hole $S$. Recall from physics that $d=v \cdot t$. So the change in volume is

$$
\Delta V \approx S v(h) \Delta t
$$

Therefore, $A(h) \Delta h \simeq S v(h) \Delta t$ by our previous observations of $\Delta V$. Rearranging and differentiating with respect to $t$ gives us the differential equation for the change in the liquid height as a function of time

$$
h^{\prime}=-\frac{S}{A(h)} v(h) .
$$

We proceed in the next subsection to use laws of physics to gain greater insight into the emptying velocity function $v(h)$.

2.1. Torricelli's Law. The liquid leaving the tank during the time $\Delta t$ will have some mass $\Delta m$. This results in a potential energy loss of $\Delta \Pi=\Delta(m g h)$ where $g$ is the acceleration due to gravity. Also, the kinetic energy of the liquid flowing out of the tank during the time $\Delta t$ is $\Delta K=\Delta\left(m v^{2} / 2\right)$. Using conservation of energy and setting $\Delta \Pi$ and $\Delta K$ equal to each other gives us

$$
v(h)=\sqrt{2 g h} .
$$

Torricelli was the person who introduced (3) to the world back in 1640 [5].

In reality, due to the viscosity of the liquid within the water tank, (3) is not accurate enough for us to use. Through experiments

$$
v(h)=\alpha \sqrt{g h},
$$

where the constant $\alpha$ depends on the physical properties of the liquid (e.g., for water $\alpha=0.84$ ) [8]. Let $\Delta V=V$ and $\Delta t=T^{*}$ in (1) and assume the liquid is flowing from the tank at the constant initial rate $v_{0}=v(H)=\alpha \sqrt{g H}$. Then the theoretical emptying time $T^{*}$ would be

$$
T^{*}=\frac{V}{S v_{0}}=\frac{V}{S \alpha \sqrt{g H}} .
$$

However, according to Torricelli's law (3), the outflow rate is decreasing with the decrease of height of the liquid. Thus the true emptying time $T$ will increase by some 
coefficient $k$ as the height of the liquid decreases. Therefore, the true emptying time $T$ is

$$
T=k T^{*}=k \frac{V}{S \alpha \sqrt{g H}},
$$

where $k>1$. In general, the coefficient $k$ depends on the height $H$ and the shape of the tank. Since the shape affects the value of $k$, we will focus on various shapes of tanks. In the proceeding sections, $k$ will be a constant, since it is defined as the ratio of $T / T^{*}$.

2.2. Emptying Time. Plugging in the velocity from Torricelli's Law (4) into the differential equation (2) gives the change in height as

$$
h^{\prime}=-S \alpha \sqrt{g} \frac{\sqrt{h}}{A(h)} .
$$

Our differential equation must satisfy two boundary conditions. The first being at $t=0$, the height of the liquid $h$ is equal to the height of the tank $H$. The second is that at the empty time $T$, there is no water left in the tank, i.e. $h=0$. After separating the variables and integrating, we obtain the implicit solution

$$
\int_{h}^{H} \frac{A(u)}{\sqrt{u}} d u=S \alpha \sqrt{g} t
$$

Notice (8) satisfies $h(0)=H$. As $h(T)=0$, we obtain from (8) that

$$
T=\frac{1}{S \alpha \sqrt{g}} \int_{0}^{H} \frac{A(h)}{\sqrt{h}} d h .
$$

Next, we want find an expression for efficiency constant $k$, implicitly defined in (6). First note that the integral of the area of a shape along its height is the volume. So

$$
V=\int_{0}^{H} A(h) d h .
$$

By equating (6) and (9) as well as using (10), we derive an expression for $k$

$$
k=k(H)=\sqrt{H} \frac{\int_{0}^{H}(A(h) / \sqrt{h}) d h}{\int_{0}^{H} A(h) d h} .
$$

Next, we want to find a general area formula for a given tank. We rotate the graph of a non-negative continuous function $f(h), 0 \leq h \leq H$, about the $h$ axis to obtain

$$
A(h)=\pi f^{2}(h) .
$$

Notice from (11) that $k$ depends on $A$ and from (12) $A$ depends on $f$. This leads to the important result that in this case the coefficient $k$ depends only on $f$. In other words, $k$ is only dependent on the shape of the tank. We will compute the coefficient $k$ for a few simple and widely used tank shapes and then use those emptying efficiency in formula (6) to obtain the emptying time. In the proceeding sections we now consider some basic shapes for water tanks and compute the corresponding emptying efficiency. 
2.2.1. Cylinder. First consider a cylindrical water tank often referred to as a drum.

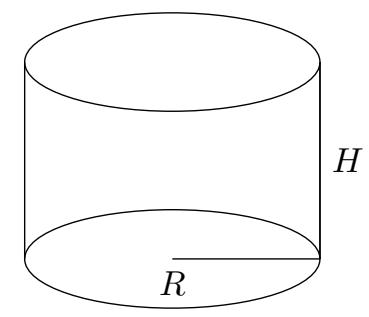

Let the cylinder tank have a height $H$ with base radius $R$, where $R^{2}=V / \pi H$. Then, $f(h)=R, 0 \leq h \leq H$. Thus, $A(h)=\pi R^{2}$. So by (11),

$$
k=\frac{\sqrt{H} \int_{0}^{H} \pi R^{2} h^{-1 / 2} d h}{\int_{0}^{H} \pi R^{2} d h}=\frac{\left.\sqrt{H} 2 h^{1 / 2}\right|_{0} ^{H}}{\left.h\right|_{0} ^{H}}=2 .
$$

2.2.2. Cone. Next, we consider a cone-shaped tank.

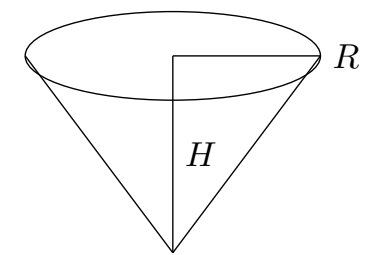

Let a cone- or funnel-shaped tank have a height $H$ and radius $R$, where $R^{2}=3 \mathrm{~V} / \pi H$. So $f(h)=\gamma h$ with $\gamma=H / R$. We have $A(h)=\pi f^{2}(h)=\pi \gamma^{2} h^{2}$. From (11),

$$
k=\frac{\sqrt{H} \int_{0}^{H} \pi \gamma^{2} h^{3 / 2} d h}{\int_{0}^{H} \pi \gamma^{2} h^{2} d h}=1.2
$$

2.2.3. Frustum of a cone. Next, consider the frustum of a cone.

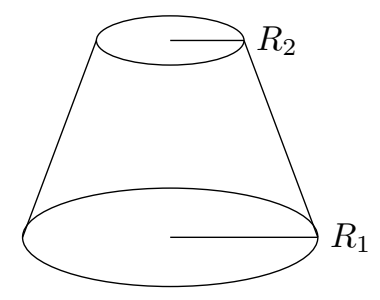


Let a frustum of a cone have a lower base radius $R_{1}$ and upper base radius $R_{2}$. Then $f(h)=a+b h$, where $a=R_{1}$ and $b=\left(R_{2}-R_{1}\right) / H$, and $A(h)=\pi(a+b h)^{2}$. Thus,

$$
\begin{aligned}
& k=\frac{\sqrt{H} \int_{0}^{H} \pi\left(a^{2} h^{-1 / 2}+2 a b h^{1 / 2}+b^{2} h^{3 / 2}\right) d h}{\int_{0}^{H} \pi\left(a^{2}+2 a b h+b^{2} h^{2}\right) d h} \\
& k=\frac{2 a^{2} H+(4 / 3) a b H^{2}+(2 / 5) b^{2} H^{3}}{a^{2} H+a b H^{2}+(1 / 3) b^{2} H^{3}} \\
& k=\frac{2 R_{1}^{2}+(4 / 3) R_{1}\left(R_{2}-R_{1}\right)+(2 / 5)\left(R_{2}^{2}-2 R_{1} R_{2}+R_{1}^{2}\right)}{R_{1}^{2}+R_{1}\left(R_{2}-R_{1}\right)+(1 / 3)\left(R_{2}^{2}-2 R_{1} R_{2}+R_{1}^{2}\right)} .
\end{aligned}
$$

Further simplification gives us

$$
k=\frac{2}{5} \frac{8 R_{1}^{2}+4 R_{1} R_{2}+3 R_{2}^{2}}{R_{1}^{2}+R_{1} R_{2}+R_{2}^{2}} .
$$

Notice all the $H$ 's cancel out. Thus $k$ is independent of $\mathrm{H}$. When $R_{1}=0$, we get a cone shape tank where $k=1.2$. For $R_{2}=0$, we obtain an inverse cone in which $k=3.2$. When $R_{1}=R_{2}$, we get a cylinder with $k=2$. The only new information learned here was the $k$ value for the inverse cone.

2.2.4. Spherical tanks. Finally, we consider a spherical tank.

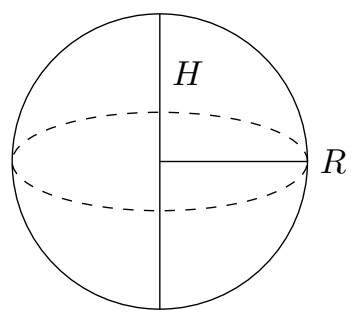

Let the spherical tank of height $H$ and radius $R$ have a volume $V=\pi H^{2}(R-H / 3)$. Then $f^{2}(h)=h(2 R-h), 0 \leq h \leq H$. Hence $A(h)=\pi h(2 R-h)$. Thus,

$$
k(H)=\frac{\sqrt{H} \int_{0}^{H}\left(2 R \pi h^{1 / 2}-\pi h^{3 / 2}\right) d h}{\int_{0}^{H}(2 R \pi h-\pi h) d h}=\frac{(4 / 3) R-(2 / 5) H}{R-(1 / 3) H}=\frac{2}{5} \frac{10 R-3 H}{3 R-H} .
$$

Since the height of a sphere equals its diameter, we have $H=2 R$ for a spherical tank. We obtain $k=1.6$. Then $H=R$ for a hemispherical tank because a hemisphere is half a sphere. We find that $k=1.4$. The table below summarizes our results of the emptying efficiency for the previous tanks. The cone shaped tank turns out to be the most efficient. This explains why funnels are cone-shaped to allow for the fastest emptying time. Formula (6) and the table below allows one to compare emptying times of tanks of various shapes with variable volume and height.

For physical reasons, the coefficient $k$ is always larger than 1 . This is because the outflow rate cannot be faster than the input rate. We can think of energy with a simple machine. When we input energy into a machine we cannot extract more 
TABLE 1

Emptying Efficiencies of Various Tanks

\begin{tabular}{|l|l|l|l|l|l|}
\hline Tank Shape & Cone & Hemisphere & Sphere & Cylinder & Inverse Cone \\
\hline$k$ & 1.2 & 1.4 & 1.6 & 2 & 3.2 \\
\hline
\end{tabular}

energy out of the machine than we put in. This fact is an application of the law of conservation of energy and a result of the first law of thermodynamics. In the next section, we will next explore if there is another shape, possibly a composite, that gives us a $k<1.2$. If we can, what is the smallest value can we achieve? This in turn would indicate the optimal shape for a water tank.

2.3. Are there Tanks with the Minimal Emptying Time? To answer the question if a minimal emptying time exists, we start with the function for the shape of a circularly symmetric tank from Hanin [8]. This general function takes the form

$$
f(h)=C h^{\mu}, \quad 0 \leq h \leq H,
$$

with some constants, $\mu \geq 0$ and $C>0$. Given $\mu$, the value of $C$ can be found from the volume equation $V=\pi C^{2} H^{2 \mu+1} /(2 \mu+1)$. From (12) and (14) we find the area $A(h)=\pi C^{2} h^{2 \mu}$. Using (11) we obtain

$$
k=\frac{\int_{0}^{H}\left(\pi C^{2} h^{2 \mu-\frac{1}{2}}\right) d h}{\int_{0}^{H}\left(\pi C^{2} h^{2 \mu}\right) d s}=\sqrt{H} \frac{2 \mu+1}{2 \mu+\frac{1}{2}} \frac{\left.h^{2 \mu+\frac{1}{2}}\right|_{0} ^{H}}{\left.h^{2 \mu+1}\right|_{0} ^{H}}=\frac{4 \mu+2}{4 \mu+1} .
$$

For $\mu=0$ and $\mu=1$, we find $k=2$ for the cylinder and $k=1.2$ for the cone, respectively. For $\mu=2$, we have $k=10 / 9$, which is a parabolic tank.

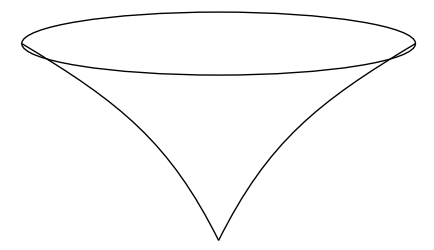

Hence, we can get a $k$ lower than 1.2. If we take the limit as $\mu \rightarrow \infty$ of (15) and using L'Hôpital's rule we get $k=1$. However, this is a theoretical minimum. Therefore, the minimum of $k=1$ cannot be practically realized as the absolute minimal emptying time. Let us see graphically what happens as $\mu$ becomes large. The volume and height of the water tower are held constant. To obtain greater understanding of the effect on the shape of changing $\mu$, we take $H=60$ and $V=100,000$ while varying $\mu$. We vary $0 \leq \mu \leq 100$ and $0 \leq h \leq H$. Next, we solve for $C$ and $f(h)$ respectfully. Notice $h$ is represented by the vertical axis and $f(h)$ is represented by the horizontal axis. 


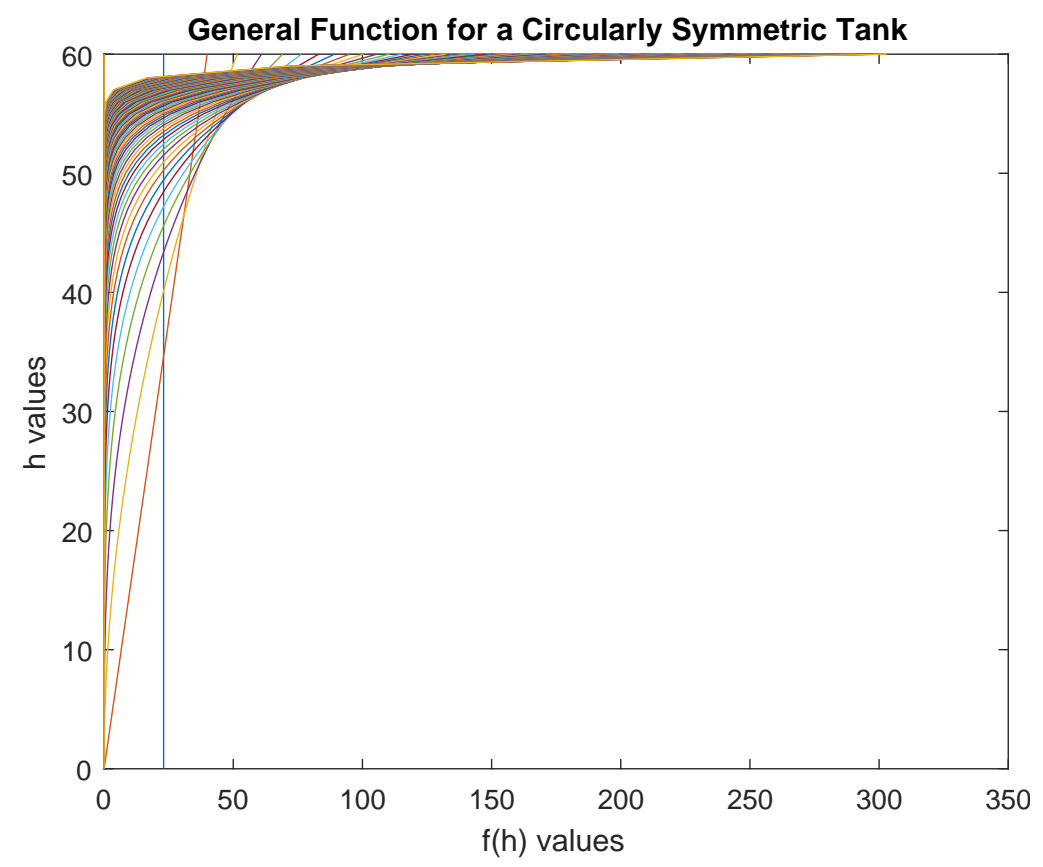

As $\mu$ becomes large, the function gets closer to the $h$ axis. We rotate the graph about the $h$ axis to obtain a vertical horn or trumpet with the bell pointed up. Theoretically, this is the most optimal shape tank.

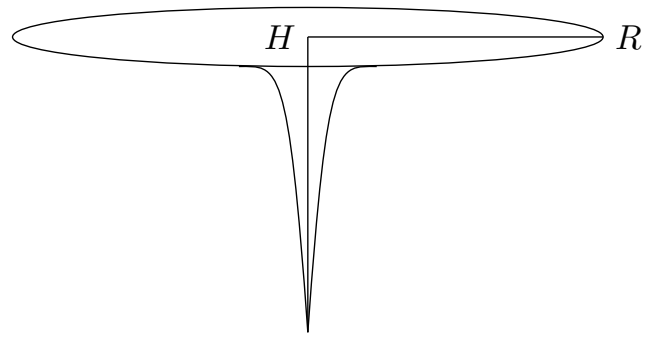

In the next section we explore how composites tanks formed by combining the basic shapes investigated above can help one get as close as possible to an optimal emptying time. From (15) we can see that there are many possible shapes based on $\mu$. It does not, however, consider the combination of different shaped tanks as one can observe in the real world. 


\section{Results on Composite Tanks.}

\subsection{Composite Tank 1.}

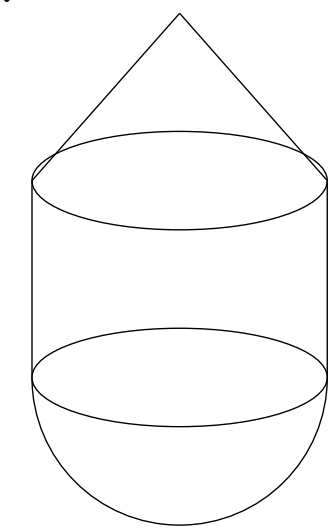

Now we will explore composite tank shapes that can readily be observed in local communities. The first tank under consideration consists of a hemisphere at the bottom, a cylinder in the middle, and an inverse cone at the top. To calculate the emptying efficiency, we can use a piecewise fuction $f(h)$ from the previous tanks. Thus $f^{2}(h)=h(2 R-h), 0 \leq h \leq \frac{1}{3} H, f(h)=R, \frac{1}{3} H<h \leq \frac{2}{3} H$, and $f(h)=a+b h$, $\frac{2}{3} H<h \leq H$. Hence the cross-sectional area is

$$
A(h)= \begin{cases}\pi h(2 R-h), & \text { if } \quad 0 \leq h \leq \frac{1}{3} H \\ \pi R^{2}, & \text { if } \quad \frac{1}{3} H<h \leq \frac{2}{3} H \\ \pi(a+b h)^{2}, & \text { if } \quad \frac{2}{3} H<h \leq H\end{cases}
$$

Then by (11),

$$
\begin{aligned}
k(H) & =\frac{\sqrt{H} \int_{0}^{\frac{1}{3} H}\left(2 R \pi h^{1 / 2}-\pi h^{3 / 2}\right) d h+\sqrt{H} \int_{\frac{1}{3} H}^{\frac{2}{3} H}\left(\pi R^{2} h^{-1 / 2}\right) d h+\sqrt{H} \int_{\frac{2}{3} H}^{H} \pi\left(a^{2} h^{-1 / 2}+2 a b h^{1 / 2}+b^{2} h^{3 / 2}\right) d h}{\int_{0}^{\frac{1}{3} H}(2 R \pi h-\pi h) d h+\int_{\frac{1}{3} H}^{\frac{2}{3} H}\left(\pi R^{2}\right) d h+\int_{\frac{2}{3} H}^{H} \pi\left(a^{2}+2 a b h+b^{2} h^{2}\right) d h} \\
& =\frac{k_{1}}{k_{2}}
\end{aligned}
$$

where

$$
\begin{aligned}
k_{1}= & \left(\frac{4}{3}\right) \frac{1}{3}^{\frac{3}{2}} R H-\left(\frac{2}{5}\right) \frac{1}{3}^{\frac{5}{2}} H^{2}+2\left(\frac{2}{3}^{\frac{1}{2}}-\frac{1}{3}^{\frac{1}{2}}\right) R^{2}+2\left(1-\frac{2}{3}^{\frac{1}{2}}\right) R_{1}^{2}+\frac{4}{3}\left(1-\frac{2}{3}^{\frac{3}{2}}\right)\left(R_{1} R_{2}-R_{1}^{2}\right) \\
& +\frac{2}{5}\left(1-\frac{2}{3}^{\frac{5}{2}}\right)\left(R_{2}^{2}-2 R_{2} R_{1}+R_{1}^{2}\right)
\end{aligned}
$$

and

$$
k_{2}=\frac{1}{9} R H-\frac{1}{81} H^{2}+\frac{1}{3} R^{2}+\frac{1}{3} R_{1}^{2}+\left(1-\frac{2}{3}^{2}\right)\left(R_{1} R_{2}-R_{1}^{2}\right)+\frac{1}{3}\left(1-\frac{2}{3}^{3}\right)\left(R_{2}^{2}-2 R_{2} R_{1}+R_{1}^{2}\right) .
$$

We want an inverse cone. So, let $R_{2}=0$. A hemispere has a radius of $R$; let $H=R$. Also, we need the radius of the bottom circle of the cone be the same has the hemisphere and cylinder. Let $R_{1}=R$. Using the above conditions we get a emptying efficiency of $k \approx 1.63$. The emptying efficient for composite tank 1 is smaller than the emptying efficient for the cylinder and inverse yet bigger than the hemisphere. Let us try to improve on the efficiency by removing the tank shape with the highest $k$ value, the inverse cone. 


\subsection{Composite Tank 2.}

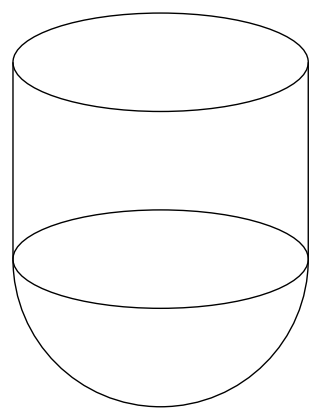

Now consider another composite tank where we have a hemisphere at the bottom and a cylinder above. Similar to composite tank 1 , we can compute the emptying efficiency with a piecewise function. So $f^{2}(h)=h(2 R-h), 0 \leq h \leq \frac{1}{2} H, f(h)=R, \frac{1}{2} H<h \leq H$. Thus we obtain the cross-sectional area

$$
A(h)=\left\{\begin{array}{lll}
\pi h(2 R-h), & \text { if } \quad 0 \leq h \leq \frac{1}{2} H \\
\pi R^{2}, & \text { if } \quad \frac{1}{2} H<h \leq H
\end{array} .\right.
$$

Then by (11),

$$
\begin{aligned}
k(H) & =\frac{\sqrt{H} \int_{0}^{\frac{1}{2} H}\left(2 R \pi h^{1 / 2}-\pi h^{3 / 2}\right) d h+\sqrt{H} \int_{\frac{1}{2} H}^{H}\left(\pi R^{2} h^{-1 / 2}\right) d h}{\int_{0}^{\frac{1}{2} H}(2 R \pi h-\pi h) d h+\int_{\frac{1}{2} H}^{H}\left(\pi R^{2}\right) d h} \\
& =\frac{\left(\frac{4}{3}\right)\left(\frac{1}{8}\right)^{\frac{1}{2}} R H-\left(\frac{2}{5}\right)\left(\frac{1}{32}\right)^{\frac{1}{2}} H^{2}+2 R^{2}+2\left(\frac{1}{2}\right)^{\frac{1}{2}} R^{2}}{\left(\frac{1}{4}\right) R H-\left(\frac{1}{24}\right) H^{2}+\left(\frac{1}{2}\right) R^{2}}
\end{aligned}
$$

A hemispere has a radius of $R$; let $H=R$. Hence we get a emptying efficiency of $k \approx 1.39$. The emptying efficiency for composite tank 2 is smaller than the emptying efficiency for composite tank 1. Interestingly, composite tank 2 has approximately the same efficiency as a hemisphere. However, the tank shape with the most efficient emptying time is still the cone.

\subsection{Composite Tank 3.}

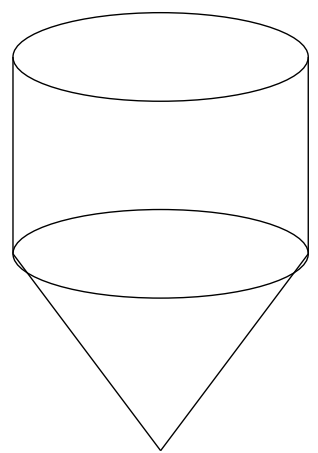

Composite tank 3 is constructed with a cone making up the bottom half and cylinder making up the top half. Admittedly, composite tank 3 does not seem common as the others, however, it should be considered as the cone has the best emptying efficiency of all the conic-shaped tanks. By construction, $f(h)=R, 0 \leq h \leq \frac{1}{2} H$ and $f(h)=\gamma h, \frac{1}{2} H<h \leq H$ where $\gamma=H / R$. Hence, we obtain the cross-sectional area

$$
A(h)=\left\{\begin{array}{ll}
\pi \gamma^{2} h^{2}, & \text { if } \quad 0 \leq h \leq \frac{1}{2} H \\
\pi R^{2}, & \text { if } \quad \frac{1}{2} H<h \leq H
\end{array} .\right.
$$


By (11),

$$
\begin{aligned}
k(H) & =\frac{\sqrt{H} \int_{0}^{\frac{1}{2} H}\left(\pi \gamma^{2} h^{3 / 2}\right) d h+\sqrt{H} \int_{\frac{1}{2} H}^{H}\left(\pi R^{2} h^{-1 / 2}\right) d h}{\int_{0}^{\frac{1}{2} H}\left(\pi \gamma^{2} h^{2}\right) d h+\int_{\frac{1}{2} H}^{H}\left(\pi R^{2}\right) d h} \\
& =\frac{\left(\frac{2}{5}\right)\left(\frac{1}{2}\right)^{\frac{5}{2}} H^{5} / R^{2}+2\left(1-\left(\frac{1}{2}\right)^{\frac{1}{2}}\right) R^{2} H}{\left(\frac{1}{2}\right)^{4} H^{5} / R^{2}+\left(\frac{1}{2}\right) R^{2} H}
\end{aligned}
$$

Let $H=R$. Then $k \approx 1.17$. Composite tank 3 has the best emptying efficiency of all the tanks considered. For composite tank 2, adding the cylinder to the hemisphere had virtually no improvement on the empty efficiency. For composite tank 3, adding the cylinder to the cone had minor improvement of about 0.03 or $2.5 \%$.

3.4. Compare tanks with example. A natural question to ask at this point is why don't we see more cone shaped tanks? They clearly have the optimal emptying efficiency according to the mathematical model. We will explore this question by starting with an example. First, we need to calculate the volume and surface area of the composite tanks.

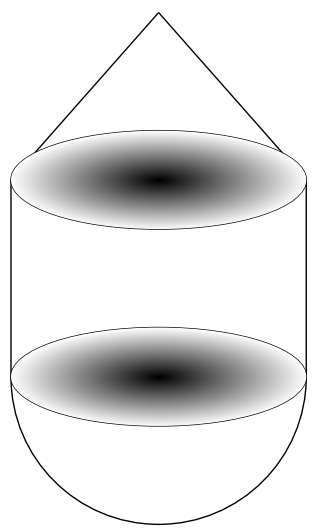

We want composite tank 1 to be at a height $2 R$ to be consistent with the other tanks and so we let $H=(2 / 3) R$ for each individual tank that makes up our composite tank to achieve our desired height. Note that we will have to adjust the hemisphere's height by multiplying the volume and area by $\frac{2}{3}$.

$$
\begin{array}{ll}
V_{\text {cone }}=\frac{1}{3} \pi R^{2} H \longrightarrow \frac{2}{9} \pi R^{3} & S A_{\text {cone }}=\pi R\left(R+\sqrt{R^{2}+H^{2}}\right) \longrightarrow\left(1+\sqrt{\frac{13}{9}}\right) \pi R^{2} \\
\frac{2}{3} V_{\text {hemi }}=\frac{2}{3} \cdot \frac{2}{3} \pi R^{3} \longrightarrow \frac{4}{9} \pi R^{3} & \frac{2}{3} S A_{\text {hemi }}=\frac{2}{3} 2 \pi R^{2} \longrightarrow \frac{4}{3} \pi R^{2} \\
V_{\text {cylinder }}=\pi R^{2} H \longrightarrow \frac{2}{3} \pi R^{3} & S A_{\text {cylinder }}=2 \pi R(R+H) \longrightarrow \frac{10}{3} \pi R^{2}
\end{array}
$$

We add the volumes of the individual tanks to obtain the volume of our composite tank (below). An interesting note is that the volume equation for the composite tank is the same as the volume equation for a sphere. We cannot simply add the surface areas of each individual tank shape to obtain the surface area of the composite tank like we did with the volume. The reason is we have extra surface area that should not be there namely circles inside the composite tank (see picture above). For example, when we computed the surface area of the cylinder we counted the circles on the top and the bottom of it. We can see this as $2 \pi R^{2}$ in the surface area of the cylinder. Turns out we have four circles we must subtract from the surface area of the composite tank. We get two circles from the cylinder, one from the cone, and one from the hemisphere. Hence, adding the surface areas of the cylinder, cone, and hemisphere as well as subtracting the area of the four circles (done above) gives us the surface area of the composite tank

$$
V_{\text {composite1 }}=\frac{4}{3} \pi R^{3} \quad S A_{\text {composite1 }}=\frac{5+\sqrt{13}}{3} \pi R^{2} .
$$


Similarly, we want composite tank 2 to be at a height $2 R$ so we let $H=R$ for each individual piece that make up our composite tank to achieve our desired height. This works out well since a hemisphere's height is naturally $R$.

$$
\begin{array}{ll}
V_{\text {hemi }}=\frac{2}{3} \pi R^{3} \rightarrow \frac{2}{3} \pi R^{3} & S A_{\text {hemi }}=2 \pi R^{2} \rightarrow 2 \pi R^{2} \\
V_{\text {cylinder }}=\pi R^{2} H \rightarrow \pi R^{3} & S A_{\text {cylinder }}=2 \pi R(R+H) \rightarrow 4 \pi R^{2}
\end{array}
$$

We add the volumes of the individual tanks to achieve the volume of our composite tank (below). Similar to the first composite tank, we must remove the unwanted area of a circle from the hemisphere and from the cylinder (see picture below). Thus, to obtain the surface area of the composite tank we subtract the area of the two circles $2 \pi R^{2}$ from the surface area of each piece.

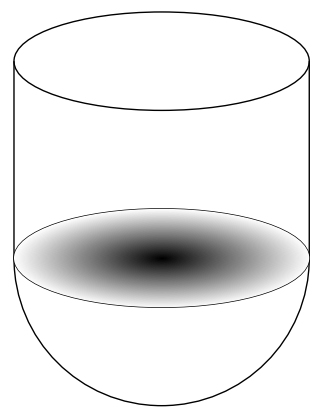

$$
V_{\text {composite } 2}=\frac{5}{3} \pi R^{3} \quad S A_{\text {composite } 2}=4 \pi R^{2}
$$

By a similar process we find that the volume and surface area of composite tank 3 are

$$
V_{\text {composite3 }}=\frac{4}{3} \pi R^{3} \quad S A_{\text {composite3 }}=(3+\sqrt{3}) \pi R^{2} .
$$

Now we look at the volume and surface area of the cone, sphere, and cylinder shaped tanks. Since we want all the tanks to have the same height, we will let the height of each tank be $2 R$. This works well because the sphere's height is naturally $2 R$. Then we obtain new volume and area equations for our tanks [1].

$$
\begin{array}{ll}
V_{\text {cone }}=\frac{1}{3} \pi R^{2} H \rightarrow \frac{2}{3} \pi R^{3} \cdot 1, & S A_{\text {cone }}=\pi R\left(R+\sqrt{\left(R^{2}+H^{2}\right)}\right) \rightarrow \pi R^{2} \cdot(1+\sqrt{5}) \\
V_{\text {sphere }}=\frac{4}{3} \pi R^{3} H \rightarrow \frac{2}{3} \pi R^{3} \cdot 2, & S A_{\text {sphere }}=4 \pi R^{2} \rightarrow 2 \pi R^{2} \cdot 2 \\
V_{\text {cylinder }}=\pi R^{2} H \rightarrow \frac{2}{3} \pi R^{3} \cdot 3, & S A_{\text {cylinder }}=2 \pi R(R+H) \rightarrow 2 \pi R^{2} \cdot 3
\end{array}
$$

A water tower is only useful if it has enough supply of water for the area it governs. Hence, we will use the fact that a typical water tank holds $1,000,000$ gallons. Let $V=1,000,000$ gallons or $V=133,680.556$ cubic feet. We first solve for the required radius $R$ in each volume equation in order for the tank to hold the given volume. Then we find the surface area $S A$ for each tank with the newly acquired radii.

\begin{tabular}{|l||l|l|}
\hline Shape & $R(\mathrm{ft})$ & \multicolumn{1}{|c|}{$S A\left(\mathrm{ft}^{2}\right)$} \\
\hline \hline Cone & 39.96 & 16,237 \\
\hline Sphere & 31.72 & 12,643 \\
\hline Cylinder & 27.71 & 14,473 \\
\hline Composite 1 & 31.72 & 9,067 \\
\hline Composite 2 & 29.45 & 10,896 \\
\hline Composite 3 & 31.72 & 14,957 \\
\hline
\end{tabular}

Observe that the sphere has the smallest surface area of the original three tanks. So the spherical tank will need less material for being built. Thus, the spherical tank will cost less than the other original two tanks to be built even though it is less efficient. This makes sense since spherical tanks are fairly common, but they are not the only ones used. The composite tanks are much more common due to the low surface area and high efficiency. The cone-shaped tank is most likely used very little because it has a high surface area making the building cost higher than the other shapes. 
3.5. Computing emptying time $\mathbf{T}$ for different tanks. Let us look at the emptying times for these tanks. We will use the emptying time equation (6)

$$
T=k \frac{V}{S \alpha \sqrt{g H}} .
$$

Let $V=133,680.556 \mathrm{ft}^{3}, H=2 R, g=32.174 \mathrm{ft} / \mathrm{s}^{2}, \alpha=0.84, S=1 \mathrm{ft}^{2}$. Then we obtain the following.

\begin{tabular}{|l||l|l|l|l|}
\hline Shape & $k$ & $R(\mathrm{ft})$ & $T(\mathrm{~s})$ & $T(\mathrm{hrs})$ \\
\hline \hline Cone & 1.2 & 39.96 & 3,766 & 1.04 \\
\hline Sphere & 1.6 & 31.72 & 5,636 & 1.56 \\
\hline Cylinder & 2.0 & 27.71 & 7,538 & 2.09 \\
\hline Composite 1 & 1.63 & 31.72 & 5,742 & 1.59 \\
\hline Composite 2 & 1.39 & 29.45 & 5,118 & 1.42 \\
\hline Composite 3 & 1.17 & 31.72 & 4,121 & 1.14 \\
\hline
\end{tabular}

Consider the cone, cylinder, and sphere. Given the same volume, the emptying time $T$ will follow the same pattern ( $\left.T_{\text {cone }}<T_{\text {sphere }}<T_{\text {cylinder }}\right)$ as the emptying efficiencies $\left(k_{\text {cone }}<k_{\text {sphere }}<k_{\text {cylinder }}\right)$. This pattern also holds for any $R_{\text {cylinder }}=R_{\text {sphere }}=R_{\text {cone }}$. To see this we first look at the denominator of the right hand side of the formula of $T$, which is the same for all the tanks. Thus, the only difference is the numerator. With the 1:2:3 ratio of the volumes of a cone, sphere, and cylinder, we see the cone will have the smallest or fastest emptying time, then the sphere, and finally the cylinder. Observe when we add the composite tanks, we see composite tank 1 has the fifth fastest time, composite tank 2 has the third fastest time, and composite tank 3 has the second fastest emptying time. Thus, when one observes composite 1 the cone on top may serve another purpose than efficiency such as keeping rain water from collecting on top. It is interesting that even though composite tank 3 has the best efficiency the cone still has the best emptying time. The cone emptying time is approximately 0.1 hours or about 355 seconds faster than composite 3 . Clearly the only difference between the two other than the emptying efficiency is the radius. By construction, the greater radius gives the cone a greater height. Thus, the velocity of is also greater, which explains why the cone has a faster emptying time.

4. Conclusion. Overall, we have presented a formula for emptying time

$$
T=k \frac{V}{S \alpha \sqrt{g H}}
$$

based on volume, emptying efficiency, cross-sectional spout area and height first derived in [8]. To use this formula we needed to find the emptying efficiency for various tank shapes as summarized in the table below.

\begin{tabular}{|l||l|}
\hline Tank Shape & $k$ \\
\hline \hline Composite 3 & 1.17 \\
\hline Cone & 1.2 \\
\hline Composite 2 & 1.39 \\
\hline Hemisphere & 1.4 \\
\hline Sphere & 1.6 \\
\hline Composite 1 & 1.63 \\
\hline Cylinder & 2 \\
\hline Inverse Cone & 3.2 \\
\hline
\end{tabular}

We verified the emptying efficiency as well as the emptying times for the above tanks and observed that cone-shaped water tank has the best emptying time. This naturally gave rise to a question as to why we do not see cone-shaped tanks more often, but this is due to the fact that among single-shaped tanks the spherical tank is more commonly used due to its smaller surface area for the same volume capacity. Thus, a spherical tank will cost less in materials to build. However, the composite tanks are in reality the most abundant because of its small emptying efficiency along with the fact that they use little surface area requiring the least amount of material. Composite tank 1 in particular is designed to help keep rain water off the top of the tank. Further study may include expanding the range of tank shapes considered and finding the emptying time, emptying efficiency, or a focus on the cost of building the tank. Also, one could focus on what materials are required for different shaped tanks since we assumed that the same material is used for all tanks. This assumption leads to the predictions about the relation between the cost and the abundance of the tanks observed in local communities. 


\section{REFERENCES}

[1] D. C. Alexander and G. M. Koeberlein, Elementary Geometry for College Students, Cengage Learning, 2010.

[2] A. N. Angelakis And X. Y. Zheng, Evolution of water supply, sanitation, wastewater, and stormwater technologies globally, 2015.

[3] M. Brain, How water towers work, April 2000. http://people.howstuffworks.com/water.htm.

[4] P. Brown, M. Evans, D. Hunt, J. McIntosh, B. Pender, and J. Ramagge, Cones, pyramids and spheres, June 2011. http://amsi.org.au/teacher_modules/Cones_Pyramids_and_ Spheres.html.

[5] R. D. Driver, Torricelli's law: An ideal example of an elementary ode, The American Mathematical Monthly, 105 (1998), pp. 453-455.

[6] S. Ferro, How do water towers work?, June 2015. http://mentalfloss.com/article/64577/ how-do-water-towers-work.

[7] L. GegGel, How do water towers work?, March 2016. http://www.livescience.com/ 54236-how-do-water-towers-work.html.

[8] L. G. Hanin, Which tanks empty faster?, The American Mathematical Monthly, 106 (1999), pp. 943-947.

[9] J. JONES, Civilization- the basis for water science, 2004.

[10] P. S. Juuti, T. KatKo, And H. Vuorinen, Environmental history of water, IWA Publishing, 2007.

[11] R. Larson and B. H. Edwards, Calculus, Cengage Learning, 2009.

[12] H. I. Shuval, A. Adin, B. Fattal, E. Rawitz, and P. Yekutiel, Wastewater irrigation in developing countries: health effects and technical solutions., Water and Sanitation Discussion Paper Series UNDP World Bank, (1990). 


\section{Appendices}

Code.

LISTING 1

General Function for a Circularly Symmetric Tank

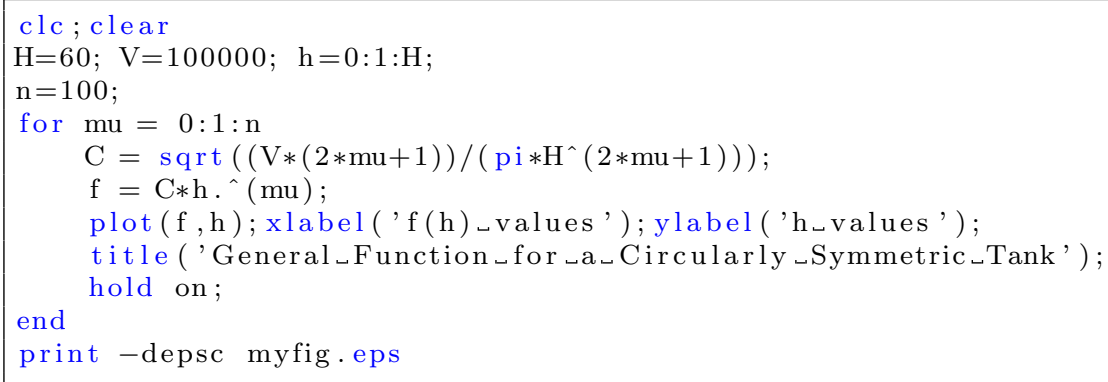

